\title{
ESTRUTURA DO DOSSEL E COMPORTAMENTO INGESTIVO DE CAPRINOS EM PASTOS DE PAPUÃ MANEJADOS SOB LOTAÇÃO CONTÍNUA
}

\section{SWARD CANOPY STRUCTURE AND INGESTIVE BEHAVIOR OF GOATS IN ALEXANDERGRASS PASTURES UNDER CONTINUOUS STOCKING}

\author{
André Brugnara Soares ${ }^{1}$ ORCID http://orcid.org/0000-0001-8328-5299 \\ Francisco Migliorini1 ${ }^{1}$ ORCID http://orcid.org/0000-0003-1064-868X \\ Christiano Santos Rocha Pitta2 ORCID http://orcid.org/0000-0002-3327-0387 \\ João Ari Gualberto Hill ${ }^{3}$ ORCID http://orcid.org/0000-0003-4175-6042 \\ André Luís Finkler da Silveira ${ }^{3}$ ORCID http://orcid.org/0000-0001-6071-5444 \\ Daniel Schmitt ${ }^{*}$ ORCID http://orcid.org/0000-0002-3679-9729
}

\author{
1 Universidade Federal Tecnológica do Paraná, Pato Branco, PR, Brasil. \\ ${ }^{2}$ Instituto Federal do Paraná, Palmas, PR, Brasil. \\ 3 Instituto Agronômico do Paraná, Pato Branco, PR, Brasil. \\ *Autor para correspondência - daniel.schmitt@veterinario.med.br
}

\section{Resumo}

O objetivo deste estudo foi descrever a estrutura do dossel e o comportamento ingestivo de caprinos em pastos de papuã manejados sob lotação contínua. Os tratamentos corresponderam a quatro alturas de manejo $(10,20,30$ e $40 \mathrm{~cm})$ mantidas através de taxa de lotação variável. As variáveis analisadas foram: composição morfológica, massa e densidade dos componentes; comprimento de lâminas foliares; valor nutritivo por simulação de pastejo; tempos de pastejo, ruminação e atividades complementares. Os dados foram submetidos à análise de variância e teste de contrastes de polinômios ortogonais. Os principais resultados foram: i) com o aumento da altura de manejo, houve um aumento da massa (total e dos componentes morfológicos) e uma inversão na composição morfológica (redução na proporção de lâminas foliares e aumento na de colmos + material morto); ii) o comprimento de lâminas foliares aumentou com a altura de manejo; iii) os animais dedicaram mais tempo ao pastejo com a redução da altura de manejo, embora aparentemente tenham abdicado do pastejo no tratamento $10 \mathrm{~cm}$ ao final do experimento. Baseado nos dados de estrutura do dossel e comportamento ingestivo, concluiu-se que pastos de papuã sob lotação contínua devem ser manejados próximos de $40 \mathrm{~cm}$. As razões são discutidas ao longo do artigo.

Palavras-chave: Brachiaria; integração lavoura-pecuária; manejo; Urochloa plantaginea; valor nutritivo.

\footnotetext{
Abstract

The aim of this trial was to describe the canopy structure and grazing behavior of goats in Alexandergrass swards under continuous stocking. The treatments corresponded to four sward canopy heights $(10,20,30$, and $40 \mathrm{~cm})$ maintained by variable stocking density. The following variables were accessed: morphological composition; nutritive value by hand plucking; mass and density of morphological components; leaf lamina length; Time spent grazing, ruminating, and idling. The data were submitted to analysis of variance and polynomial orthogonal contrasts. The main
} 
results were: i) following the sward canopy height increments, there was an increment in forage mass (and its morphological components), as well as an inversion in morphological compositions (leaf proportion reduction and stem + dead material increment); ii) leaf lamina length increased with grazing height increments; iii) time spent grazing was increased following sward canopy height reduction, although animals apparently abdicated to graze in $10 \mathrm{~cm}$ treatment at the final of the experiment. Based on sward canopy structure and grazing behavior, we concluded that Alexandergrass under continuous stocking should be managed around $40 \mathrm{~cm}$. Reason are discussed throughout the manuscript.

Keywords: Brachiaria; integrated crop-livestock system; management; nutritive value; Urochloa plantaginea.

Recebido em 15 de setembro de 2017.

Aceito em 07 de junho de 2019.

\section{Introdução}

A Urochloa (Syn. Brachiaria) plantaginea, popularmente conhecida como papuã, é uma gramínea anual de verão, de hábito de crescimento decumbente e que ocorre espontaneamente em lavouras durante o verão e início do outono. Em áreas destinadas aos sistemas integrados de produção agropecuária, o papuã pode prover forragem a um custo muito reduzido, uma vez que apresenta bom valor nutritivo, alto potencial de ressemeadura natural e desempenho animal, além de se beneficiar dos tratos culturais (por exemplo, adubação) que são despendidos durante a fase lavoura ${ }^{(1-3)}$. Adicionalmente, por estar presente em grande quantidade após a colheita da lavoura em sistemas integrados de produção agropecuária, pode ser considerada como uma alternativa para os vazios forrageiros outonais, servindo de alimento junto da palha e dos grãos que eventualmente são perdidos durante a colheita da lavoura de verão ${ }^{(1)}$.

Apesar das suas vantagens dentro de um sistema produtivo, essa forrageira ainda continua sendo pouco explorada. Uma das explicações pode ser a falta de metas de manejo eficazes para a criação e manutenção de estruturas que favoreçam o processo de forrageamento. Segundo Hodgson et al. ${ }^{(4)}$ a produtividade animal não depende apenas da produção de forragem e/ou do seu valor nutritivo, mas também de como o manejo afeta a estrutura do pasto, uma vez que isso determina a facilidade com que os animais consomem a forragem produzida. Dentro desse contexto, metas de manejo baseadas em altura têm se mostrado uma ferramenta eficaz na produção de forragem em quantidade e qualidade, além de criar estruturas que favoreçam o processo de forrageamento ${ }^{(5-6)}$.

Pastos manejados sob lotação contínua apresentam uma amplitude específica de alturas na qual podem ser mantidos sem que haja comprometimento da sua capacidade produtiva ${ }^{(6-7)}$. Embora não haja dados conclusivos na literatura científica acerca do papuã, sabe-se que espécies com estrutura e porte semelhantes (por exemplo, Brachiaria brizantha cv. Marandu) durante períodos de condições climáticas favoráveis (i.e. primavera-verão), podem ser mantidos entre $20-40 \mathrm{~cm}$ sem que haja comprometimento do valor nutritivo da forragem colhida e do processo de forrageamento ${ }^{(8)}$. Entretanto, não há relatos se essas seriam as alturas ideais para a criação e manutenção de estruturas em pastos de papuã. Dentro desse contexto, o objetivo deste trabalho é descrever a estrutura do dossel, o valor nutritivo da forragem colhida e o comportamento ingestivo de caprinos em pastos de papuã sob lotação contínua com diferentes alturas de manejo. 


\section{Material e métodos}

O experimento foi conduzido na unidade Experimental do Instituto Agronômico do Paraná (IAPAR), localizada no município de Pato Branco/PR, coordenadas de $25^{\circ} 07^{\prime}$ Sul e $52^{\circ} 41^{\prime}$ Oeste, durante os meses de janeiro e abril de 2010. Segundo o sistema de classificação de Köppen, o clima da região é do tipo Cfa - subtropical úmido ${ }^{(9)}$. Os dados referentes às condições climáticas durante o período experimental são apresentados na Figura 1.

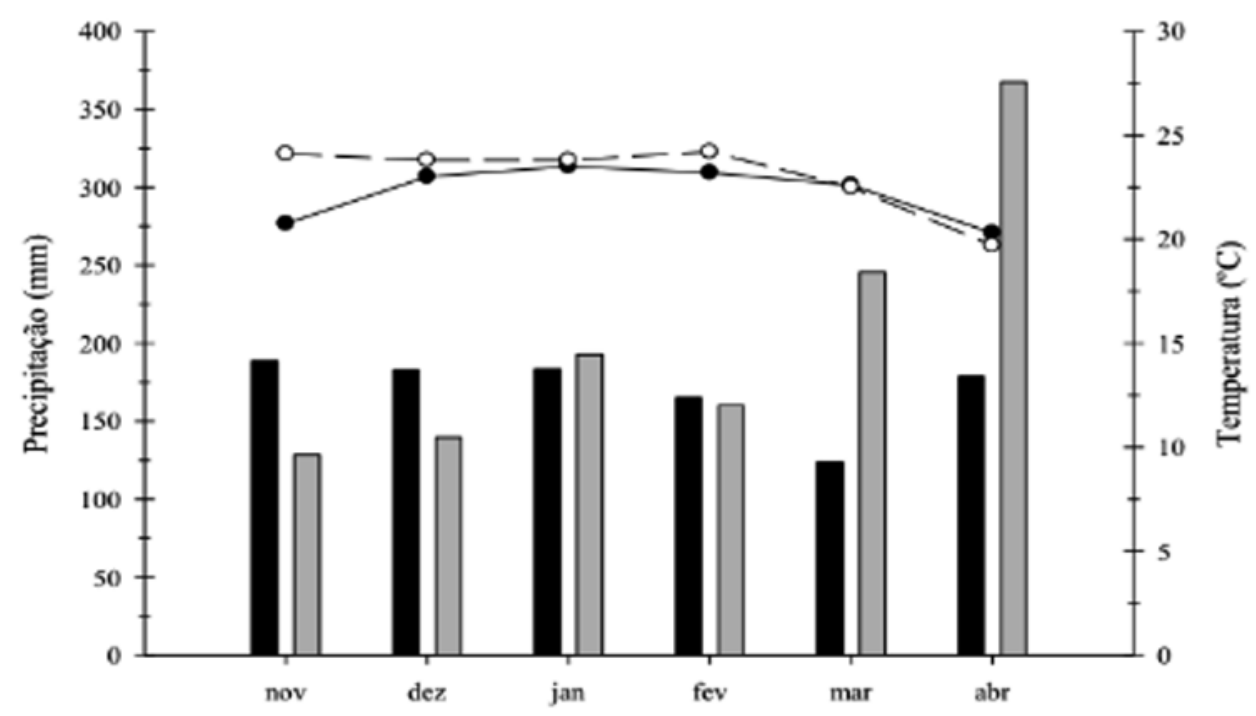

Figura 1. Dados meteorológicos históricos (1979-2009) e observados durante o período experimental (2009/2010) registrados no Instituto Agronômico do Paraná em Pato Branco/PR. Linhas representam as temperaturas médias históricas (pontilhada) e durante o período experimental (sólida). Barras representam as precipitações mensais históricas (preto) e durante o período experimental (cinza).

O solo da área experimental é classificado como Latossolo Vermelho distroférrico ${ }^{(10)}$, apresentando relevo ondulado. Antes do início do experimento, amostras de solo na camada de $0-15 \mathrm{~cm}$ foram coletadas e sua análise química apresentou os seguintes resultados: $\mathrm{pH}\left(\mathrm{CaCl}_{2}\right)$ 4,7; $\mathrm{MO}$ (digestão úmida; $\left.\mathrm{g} / \mathrm{dm}^{3}\right)$ 67,2; $\mathrm{P}$ (extrator Mehlich, $\left.\mathrm{mg} / \mathrm{dm}^{3}\right)$ 19,9; $\mathrm{K}\left(\mathrm{cmol}_{\mathrm{c}} / \mathrm{dm}^{3}\right)$ 399,7; $\mathrm{Ca}(\mathrm{KCl} 1 \mathrm{~mol} / \mathrm{L}$; $\left.\mathrm{cmol}_{\mathrm{c}} / \mathrm{dm}^{3}\right)$ 5,4; $\mathrm{Mg}\left(\mathrm{KCl} 1 \mathrm{~mol} / \mathrm{L} ; \mathrm{cmol}_{\mathrm{c}} \mathrm{dm}^{-3}\right)$ 2,7; $\mathrm{H}+\mathrm{Al}\left(\mathrm{KCl} 1 \mathrm{~mol} / \mathrm{L} ; \mathrm{cmolc}^{2} \mathrm{dm}^{3}\right)$ 7,9; CTC $\left(\mathrm{cmol}_{\mathrm{c}} / \mathrm{dm}^{3}\right)$ 16,9; SB $\left(\mathrm{cmol}_{\mathrm{c}} / \mathrm{dm}^{3}\right)$ 9,0; V (\%) 52,3. Com base na caracterização química do solo e baseado no Manual de Adubação e Calagem da Comissão de Química e Fertilidade do Solo dos Estados do Rio Grande do Sul e Santa Catarina (11), adubações foram realizadas. A adubação nitrogenada foi fracionada em três aplicações, totalizando $200 \mathrm{~kg} \mathrm{~N} / \mathrm{ha}$ na forma de ureia (45\% de $\mathrm{N}$ ), observando as condições de clima e umidade favoráveis para o máximo aproveitamento do $\mathrm{N}$ pela planta. Na primeira aplicação, utilizou-se $50 \%$ da dose total e nas demais $25 \%$ cada, nas seguintes datas: 21/12/2009, 28/01/2010 e 15/03/2010. A adubação fosfatada foi realizada uma única vez, no dia 21/12/2009, na dose $100 \mathrm{~kg} \mathrm{P}_{2} \mathrm{O}_{5} /$ ha, tendo como fonte superfosfato triplo.

O experimento foi implantado em uma área previamente utilizada $(\approx 20$ anos $)$ com integração lavourapecuária em sistema de plantio direto. A cultura antecessora foi aveia preta (Avena strigosa Scherb.) cv. IAPAR 61 consorciada com azevém (Lolium multiflorum Lam.) cv. São Gabriel. No dia 10 de dezembro de 2009, realizou-se uma roçada a fim de diminuir a cobertura vegetal sobre o solo, 
proporcionando assim uma melhor germinação das sementes de papuã (Urochloa (Syn. Brachiaria) plantaginea). O estabelecimento dos pastos de papuã foi por ressemeadura natural, pois a área possuía elevada quantidade de sementes em decorrência do diferimento realizado no ano anterior à implantação do presente trabalho.

Os tratamentos experimentais corresponderam a quatro alturas do dossel $(10,20,30$ e $40 \mathrm{~cm})$, mantidas relativamente constantes por meio da adição ou retirada de animais das unidades experimentais sempre que a altura do pasto apresentava valores acima ou abaixo da meta para cada tratamento. Portanto, durante todo o período de avaliação, adotou-se a técnica de lotação contínua com taxa de lotação variável. A escolha dessas alturas foi baseada em trabalhos realizados com Brachiaria brizantha $\mathrm{cv}$. Marandu ${ }^{(8,12)}$, por suas semelhanças estruturais e escassez de metas de altura para a cultura do papuã. As densidades médias de lotação utilizadas de cada tratamento durante o período experimental são apresentadas na Figura 2.

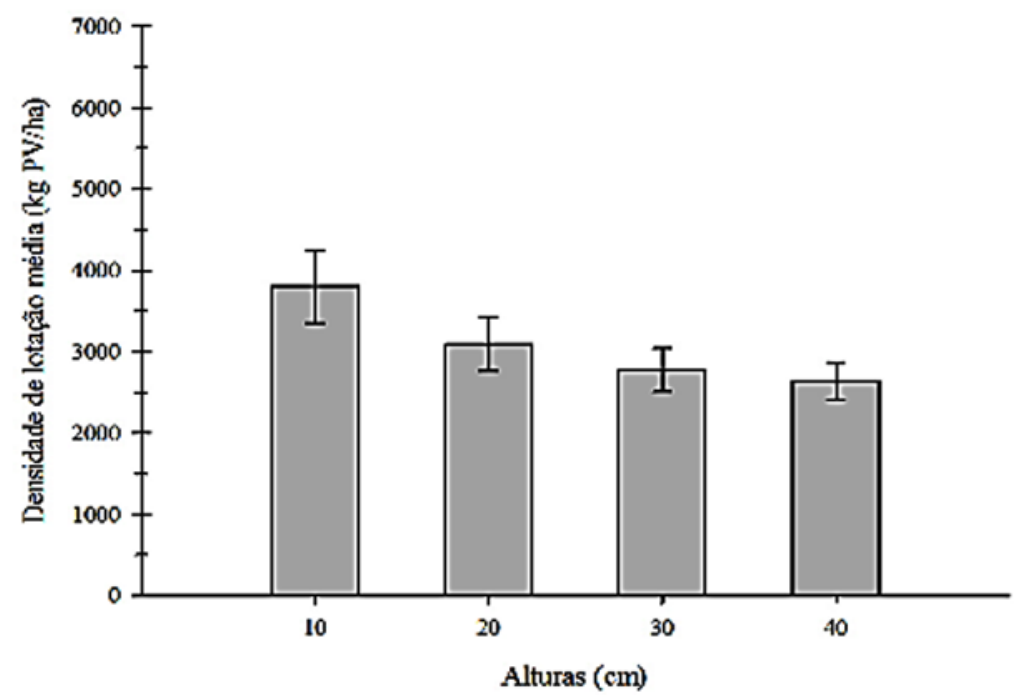

Figura 2. Densidade de lotação média utilizada nos diferentes tratamentos durante o período experimental. Barras verticais correspondem ao erro padrão da média.

O delineamento experimental adotado para a condução do experimento foi o de blocos ao acaso com três repetições. Para tanto, uma área total $(0,55 \mathrm{ha})$ foi dividida em 12 piquetes, sendo a área determinada em função dos tratamentos $(0,033$ - 0,059 ha), com o objetivo de manter um número de animais relativamente equilibrado entre os piquetes. Os animais utilizados foram cabras provenientes do Instituto Agronômico do Paraná - IAPAR - Pato Branco/PR, com média de um ano de idade e peso inicial de $38 \pm 5,3 \mathrm{~kg}$. Para um maior conforto dos animais, foram disponibilizados acesso a apriscos de $4 \mathrm{~m}^{2}$ (2m x $\left.2 \mathrm{~m}\right)$, com solado ripado, água fresca e sal mineral.

A partir de uma roçada de nivelamento efetuada no dia 10 de dezembro de 2009, iniciou-se o monitoramento da altura dos pastos em cada unidade experimental, com o auxílio de um bastão graduado, em 40 pontos aleatórios por unidade experimental. Assim que os pastos atingiram a altura média de $30 \mathrm{~cm}$ (dia 31/12/2009 - PR), o pastejo foi iniciado, ajustando-se a taxa de lotação (kg de $\mathrm{PV} / \mathrm{ha}$ ) para estabelecer as alturas pretendidas. No dia 06 de janeiro de 2010, deu-se início ao período experimental.

As avaliações de valor nutritivo foram realizadas em todas as unidades experimentais através do método de simulação manual de pastejo, conforme metodologia descrita por Euclides et al ${ }^{(13)}$. Para tanto, aproximadamente $700 \mathrm{~g}$ de massa fresca de forragem foram coletadas e levadas à estufa de 
ventilação forçada a $60^{\circ} \mathrm{C}$ durante 72 horas. Quando secas, as amostras eram trituradas em moinho tipo "Wiley", utilizando-se peneira com malha de $1 \mathrm{~mm}$. As amostras foram então analisadas através do NIRS, em que se determinou os teores (\%MS) de proteína bruta (PB), fibra insolúvel em detergente neutro (FDN) e fibra insolúvel em detergente ácido (FDA).

A quantificação dos componentes morfológicos foi realizada a partir de amostras de forragem coletadas com auxílio de armações circulares de $0,1 \mathrm{~m}^{2}$ dispostas em dois pontos representativos da condição média da unidade experimental no momento da avaliação (i.e., ao final de cada período experimental). Após coletados, esses materiais foram manualmente separados entre os componentes morfológicos: lâminas foliares, colmos (colmo verdadeiro + pseudocolmo) e material morto, acondicionados em sacos de papel e secos em estufa de circulação forçada de ar a $65^{\circ} \mathrm{C}$ durante 72 horas. Logo após, o material era pesado separadamente e utilizado para a determinação do valor percentual dos componentes morfológicos (quociente entre a massa do componente e o somatório de todos os componentes), massa de forragem (somatório da massa de todos os componentes; $\mathrm{kg} \mathrm{MS} / \mathrm{ha}$ ) e densidade volumétrica (quocientes entre a massa de forragem ou do componente morfológico e a altura do dossel; $\mathrm{kg} \mathrm{MS} / \mathrm{ha} / \mathrm{cm})$.

A avaliação de comprimento de lâmina foliar foi realizada em dois intervalos de 8 dias (Ciclo 1: 02/02/2010 a 10/02/2010; Ciclo 2: 05/03/2010 a 13/03/2010), onde, diariamente, eram efetuadas leituras do comprimento de todas as lâminas foliares de 30 perfilhos marcados em cada unidade experimental. O critério para medir o comprimento das lâminas foliares foi estabelecido de acordo com o estádio de desenvolvimento das mesmas. Se as lâminas fossem expandidas, o comprimento corresponderia ao intervalo entre a ponta até sua lígula. Quando as lâminas estavam em expansão, o mesmo procedimento foi adotado, porém, considerando a lígula da última folha expandida como referência. Quando as lâminas foliares estavam em processo de senescência, ao invés da ponta da lâmina foliar, considerou-se o ponto até onde o processo de senescência tinha avançado.

As avaliações de comportamento ingestivo foram realizadas visualmente durante dois períodos (Ciclo 1: 02/02/2010; Ciclo 2: 05/03/2010) em todas as unidades experimentais por um período de 24 horas. Para tanto, três animais foram selecionados baseados na idade e peso em cada unidade experimental, onde foram monitorados a cada 10 minutos durante suas três principais atividades: i) pastejo atividades relacionadas à procura, seleção e apreensão de forragem; ii) ruminação - movimentos mandibulares não relacionados com pastejo; iii) atividades complementares - momentos em que os animais não estavam pastejando ou ruminando (por exemplo, descansando ou bebendo água). Esses dados foram utilizados para estimar a proporção (\%) e o tempo (minutos) despendido com cada atividade. Vale ressaltar que os animais foram previamente treinados/acostumados ao manejo/presença dos avaliadores e mantidos sem suplementação durante o transcorrer do experimento.

Após coletados, os dados foram submetidos à análise de variância pelo PROC MIXED do SAS (versão 9.0) e os efeitos linear, quadrático e cúbico da altura de manejo testados por meio de contrastes de polinômios. Os períodos de avaliação foram considerados como medidas repetidas no tempo, uma vez que se buscava uma resposta padrão ao longo do ciclo de produção. Exceção se fez aos dados de comportamento ingestivo, uma vez que foi observada uma marcante diferença entre as tendências dos ciclos de avaliação. A significância adotada para todas as análises foi de $5 \%$ (P $\leq$ $0,05)$. 


\section{Resultados e discussão}

A implantação dos tratamentos, i.e., intensidade de pastejo, nas quatro alturas pretendidas $(10,20,30$ e $40 \mathrm{~cm}$ ), foram realizadas através do pastejo com caprinos. As alturas médias registradas durante todo o período experimental são apresentadas na Figura 2. O período de rebaixamento dos pastos (PR), i.e., estabelecimento das alturas pretendidas, foram programados para ter duração de 7 dias. Entretanto, para os tratamentos de 20 e $10 \mathrm{~cm}$, foi necessário utilizar parte do período 1 (P1), uma vez que foi necessário o emprego de altas severidades de desfolhação (aproximadamente $50 \%$ no tratamento $20 \mathrm{~cm}$ e $75 \%$ no tratamento $10 \mathrm{~cm}$ ), além de se optar por não comprometer o processo de forrageamento dos animais durante esse período. Segundo Fonseca et al ${ }^{(14)}$ e Mezzalira et al (15), severidades de desfolhação acima de $40 \%$ impõem restrições à formação do bocado, reduzindo a velocidade de ingestão de forragem. Ao final do período experimental (P4), as médias de altura abaixo das metas de manejo se devem ao baixo crescimento da cultura, em função do grande número de dias chuvosos e com temperatura amena (Figura 1). Entretanto, tais fatos não comprometem as respostas observadas no presente experimento, já que um contraste entre as metas de manejo foi alcançado (diferenças entre médias maiores que $7 \mathrm{~cm}$; Figura 3). Portanto, o manejo da taxa de lotação foi satisfatório para assegurar um contraste entre os tratamentos propostos.

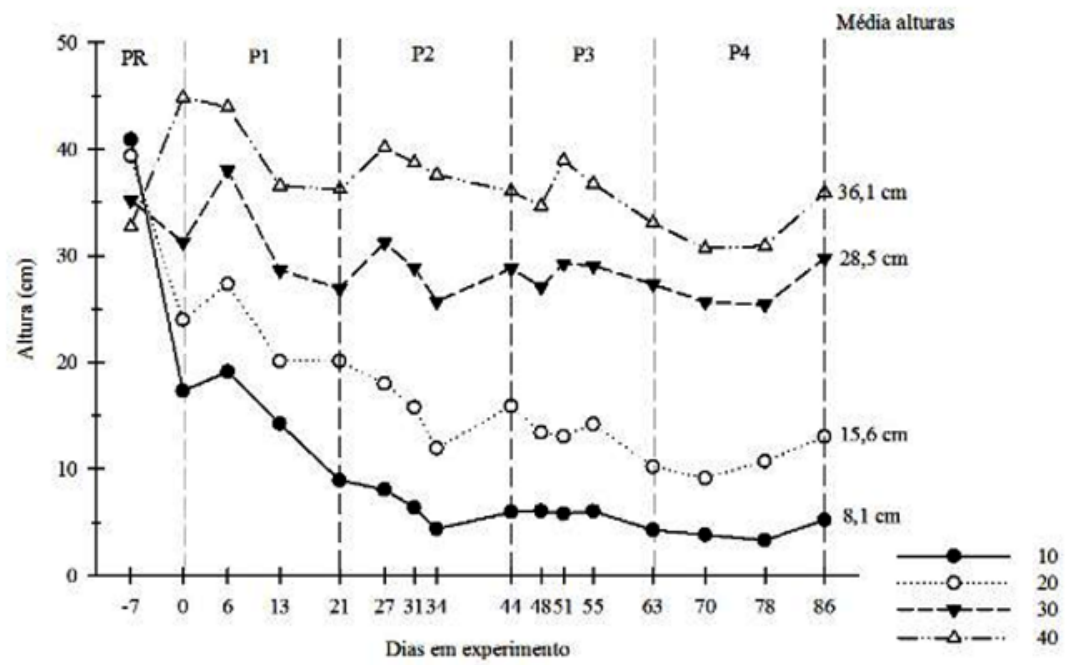

Figura 3. Dinâmica da altura de pastos de papuã $(10,20,30$ e $40 \mathrm{~cm})$ observada nos diferentes tratamentos ao longo do período experimental. Período: PR: 31/12/09 - 06/01/10; P1: 06/01/10 27/01/10; P2: 27/01/10 - 19/02/10; P3: 19/02/10 - 12/03/10; P4: 12/03/10 - 02/04/10.

As alturas de manejo afetaram as concentrações de $\mathrm{PB}(\mathrm{P}<0,0001)$ e FDN $(\mathrm{P}=0,0030)$, mas não as de FDA ( $\mathrm{P}=0,8859)$, havendo uma ligeira melhora da composição química com a diminuição da altura de manejo (Tabela 1). A explicação para tal fato já está bem estabelecida na literatura científica, uma vez que pastos manejados baixos são desfolhados com maior frequência, gerando estruturas com maior quantidade de lâminas foliares jovens e de melhor valor nutritivo ${ }^{(16-18)}$. Segundo Chapman et al ${ }^{(18)}$, lâminas foliares jovens apresentam maior concentração de carboidratos não-estruturais e enzimas e menor concentração de componentes estruturais. Adami et al ${ }^{(1)}$ em um estudo sobre a influência da combinação de duas intensidades de pastejo (1.500 ou $3.000 \mathrm{~kg}$ de $\mathrm{MS} / \mathrm{ha}$ ) e três níveis de adubação nitrogenada $(0,200$ e $400 \mathrm{~kg} \mathrm{~N} / \mathrm{ha}$ de nitrogênio na forma de ureia) sobre a dinâmica da pastagem de papuã em lotação contínua (resultando em alturas entre 16 - $39 \mathrm{~cm}$ ), não encontraram 
diferenças significativas na composição química da forragem entre os tratamentos, além de reportarem valores qualitativamente inferiores aos do presente experimento. No entanto, os resultados aqui apresentados se referem à coleta obtida através da simulação de pastejo que procura representar a seleção por lâminas foliares apresentadas aos animais e não coletando a planta inteira (i.e., rente ao solo), que possui maior proporção de colmos e material morto e, consequentemente, menor valor nutritivo. Segundo Euclides et al (13) o método de simulação de pastejo permite a avaliação da qualidade das forrageiras em pastejo com boa acurácia, desde que seja feita por avaliadores treinados. Por fim, aparentemente não há restrições de ordem química para produção animal em pastos de papuã dentro das estratégias de manejo adotadas no presente estudo, pois, ao comparar com o reportado na literatura, seriam condizentes com bons níveis de desempenho animal ${ }^{(8,19)}$.

Tabela 1. Composição química da forragem obtida por simulação de pastejo de pastos de papuã mantidos em 10, 20, 30 e $40 \mathrm{~cm}$ de altura por caprinos em lotação contínua

\begin{tabular}{|c|c|c|c|c|c|c|}
\hline \multirow{2}{*}{ Variável } & \multicolumn{4}{|c|}{ Tratamento $(\mathrm{cm})$} & \multirow{2}{*}{ EPM } & \multirow[t]{2}{*}{ Efeito } \\
\hline & 10 & 20 & 30 & 40 & & \\
\hline \multicolumn{7}{|c|}{ Composição química } \\
\hline PB & 23,8 & 22,6 & 22,0 & 21,6 & 0,25 & $\mathrm{~L}$ \\
\hline FDN & 52,6 & 53,7 & 55,0 & 55,3 & 0,52 & $\mathrm{~L}$ \\
\hline FDA & 23,1 & 23,5 & 23,1 & 23,6 & 0,57 & NS \\
\hline
\end{tabular}

Abreviações: PB (proteína bruta); FDN (fibra insolúvel em detergente neutro); FDA (fibra insolúvel em detergente ácido); L (efeito linear); NS (não significativo, P > 0,05); EPM (erro padrão da média). Unidade: \% MS.

A composição morfológica variou de forma significativa com os tratamentos, havendo um aumento linear na proporção de lâminas foliares $(\mathrm{P}=0,0174)$ e uma diminuição linear na proporção de colmos + material morto $(\mathrm{P}=0,0153)$ com a diminuição da altura de manejo (Tabela 2). Tais respostas podem ser atribuídas a: i) menor necessidade de tecidos de sustentação nos pastos manejados baixos, principalmente em função do menor comprimento das lâminas foliares (Figura 4; ${ }^{(20)}$ ); ii) menor competição por luz e, consequentemente, menores taxas de senescência e alongamento de colmos nos pastos manejados baixos ${ }^{(5)}$; iii) facilitação de colheita das lâminas foliares nos pastos manejados altos ${ }^{(8,21)}$. Cabe ressaltar que os dados aqui encontrados foram superiores (i.e., com maior proporção de lâminas foliares e menor de colmos + material morto) aos observados em outros experimentos com plantas do gênero Brachiaria, de metodologia e alturas de manejo semelhantes $(8,17,22-24)$.

Tabela 2. Composição morfológica de pastos de capim-papuã mantidos em 10, 20, 30 e $40 \mathrm{~cm}$ de altura por caprinos em lotação contínua

\begin{tabular}{|c|c|c|c|c|c|c|}
\hline \multirow{2}{*}{ Variável } & \multicolumn{4}{|c|}{ Tratamento $(\mathrm{cm})$} & \multirow{2}{*}{ EPM } & \multirow[t]{2}{*}{ Efeito } \\
\hline & 10 & 20 & 30 & 40 & & \\
\hline \multicolumn{7}{|c|}{ Composição morfológica } \\
\hline Folhas & 48,7 & 41,8 & 38,2 & 36,3 & 2,8 & $\mathrm{~L}$ \\
\hline Colmos + M. morto & 51,3 & 58,2 & 61,8 & 63,7 & 2,8 & $\mathrm{~L}$ \\
\hline
\end{tabular}

Abreviações: L (efeito linear); EPM (erro padrão da média). Unidade: \% da MS.

Houve uma redução linear da massa de forragem total e dos componentes morfológicos com a diminuição da altura de manejo ( $\mathrm{P}<0,0001$; Tabela 3). Isso ocorreu em função da crescente densidade de lotação com a diminuição da altura de manejo (Figura 2), havendo maior remoção do 
material acumulado. De certa forma, esses resultados já eram esperados, uma vez que a altura de manejo apresenta correlação positiva com a massa de forragem. Segundo Da Silva e Nascimento Jr. (25), a altura de manejo pode ser utilizada como um critério confiável de manejo para a criação de estruturas de dossel forrageiro, tornando-se uma ferramenta prática e útil em nível de campo, com boa representação e baixo custo de operação. Em comparação com outros experimentos, envolvendo o uso de pastos de Brachiaria brizantha sob lotação contínua e metas de altura semelhantes, os valores de massa aqui encontrados foram condizentes com aqueles reportados em ganhos de peso vivo de bovinos de até $930 \mathrm{~g} / \mathrm{dia}^{(8,22)}$.

Tabela 3: Massa de forragem e dos componentes morfológicos de pastos de capim-papuã mantidos em 10, 20, 30 e $40 \mathrm{~cm}$ de altura por caprinos em lotação contínua

\begin{tabular}{|c|c|c|c|c|c|c|}
\hline \multirow{2}{*}{ Variável } & \multicolumn{4}{|c|}{ Tratamento (cm) } & \multirow{2}{*}{ EPM } & \multirow[t]{2}{*}{ Efeito } \\
\hline & 10 & 20 & 30 & 40 & & \\
\hline \multicolumn{7}{|c|}{ Massa de forragem } \\
\hline Total & 853 & 1941 & 2939 & 4110 & 146 & $\mathrm{~L}$ \\
\hline Lâminas foliares & 406 & 819 & 1133 & 1524 & 118 & $\mathrm{~L}$ \\
\hline Colmos + M. morto & 447 & 1122 & 1806 & 2586 & 118 & $\mathrm{~L}$ \\
\hline
\end{tabular}

Abreviações: L (efeito linear); EPM (erro padrão da média). Unidade: kg MS/ha.

Com relação aos dados de densidade, apenas os de lâminas foliares variaram de forma significativa em função dos tratamentos ( $\mathrm{P}=0,0039$; Tabela 4). Isso sugere que os aumentos em massa de forragem e de colmos + material morto foram proporcionais aos incrementos em altura (Tabela 2), gerando valores relativamente constantes de suas densidades (Tabela 4), mas que os aumentos em massa de lâminas foliares foram menores e desproporcionais aos incrementos em altura (Tabela 2), ocasionando a redução da densidade de lâminas foliares. Logo, é provável que o crescimento dos perfilhos tenha ocorrido majoritariamente em função do alongamento/acúmulo de colmos (apresentando maiores incrementos em massa de forragem; Tabela 3) e da disposição mais ereta/vertical das lâminas foliares (apresentando menores incrementos em massa de lâminas foliares; Tabela 3). De fato, pastos em competição por luz (p. ex. altos) apresentam maiores taxas de alongamento de colmos e dispõem suas lâminas foliares de maneira vertical, maximizando assim a captação de luz em estratos superiores e de maior incidência luminosa ${ }^{(8,25)}$. Todavia, cabe ressaltar que experimentos clássicos com "micropastos" demonstram que a massa do bocado é favorecida em estruturas relativamente altas e pouco densas $(26,27)$, desde que não apresentem proporções significativas de colmos de alta resistência a tração em sua composição ${ }^{(27-29)}$. Em outras palavras, pastos baixos e densos podem implicar em dificuldades aos animais em apreender a forragem, afetando negativamente o consumo e o desempenho ${ }^{(30)}$. Sendo assim, o aumento da densidade dos pastos manejados baixos não pode ser interpretado per se como algo vantajoso para a formação do bocado e do processo de forrageamento.

Houve uma diminuição linear no comprimento médio de lâminas foliares com a diminuição da altura de manejo ( $\mathrm{P}<0,0001)$. Um dos fatores que justifica a redução do tamanho da folha com o aumento da intensidade de pastejo é o menor comprimento de bainha a ser percorrido pela folha até sua exposição ao ambiente externo ${ }^{(31)}$. Ademais, pastos manejados baixos apresentam uma maior frequência de desfolhação em nível de perfilhos individuais, já que para se manterem baixos há a necessidade de uma maior densidade de lotação ${ }^{(16,32)}$. Cabe ressaltar que o valor limítrofe para a 
formação do bocado $\left(7-10 \mathrm{~cm}^{(32-33)}\right)$ foi encontrado apenas em pastos manejados com menos de 20 $\mathrm{cm}$, indicando que para não impor restrições severas de consumo por questões estruturais, pastos de papuã sob lotação contínua devem ser manejados com mais de $20 \mathrm{~cm}$ (Figura 4).

Tabela 4. Densidade de forragem total e dos componentes morfológicos de pastos de capim-papuã mantidos em 10, 20, 30 e $40 \mathrm{~cm}$ de altura por caprinos em lotação contínua

\begin{tabular}{|c|c|c|c|c|c|c|}
\hline \multirow{2}{*}{ Variável } & \multicolumn{4}{|c|}{ Tratamento $(\mathrm{cm})$} & \multirow{2}{*}{ EPM } & \multirow[t]{2}{*}{ Efeito } \\
\hline & 10 & 20 & 30 & 40 & & \\
\hline \multicolumn{7}{|c|}{ Densidade de forragem } \\
\hline Total & 117,2 & 124,0 & 103,1 & 113,0 & 6,6 & NS \\
\hline Lâminas foliares & 64,4 & 54,4 & 39,3 & 41,1 & 5,2 & $\mathrm{~L}$ \\
\hline Colmos + M. morto & 53,7 & 71,4 & 63,8 & 71,9 & 4,9 & NS \\
\hline
\end{tabular}

Abreviações: L (efeito linear); NS (não significativo, P > 0,05); EPM (erro padrão da média). Unidade: $\mathrm{kg} \mathrm{MS} / \mathrm{ha} / \mathrm{cm}$.

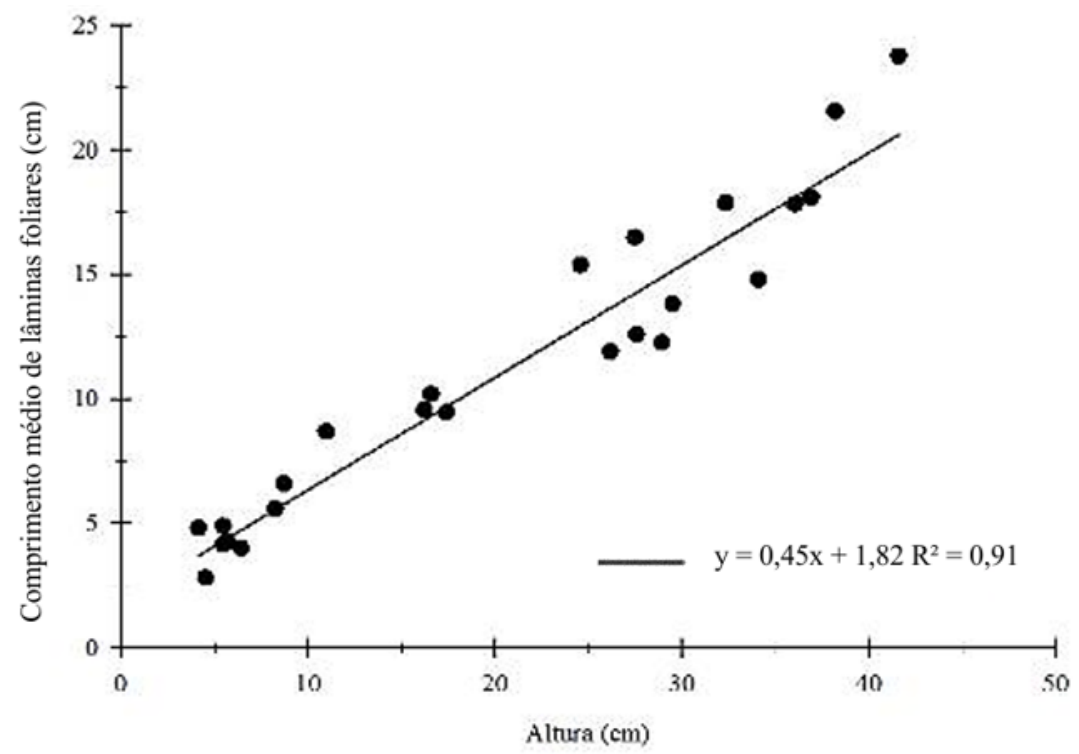

Figura 4. Relação entre o comprimento médio de lâmina foliar $(\mathrm{cm})$ e a altura do dossel forrageiro em pastos de papuã manejados sob lotação contínua com caprinos.

Os dados de comportamento ingestivo variaram significativamente em função dos tratamentos ( $\mathrm{P}<$ 0,05; Tabela 5). De modo geral, a redução da altura de manejo (40 - $20 \mathrm{~cm})$ acarretou em aumento nos tempos de pastejo e atividades complementares, bem como na diminuição do tempo de ruminação. Esses resultados, quando interpretados em conjunto, indicam uma gradativa restrição estrutural ao processo de forrageamento e uma tentativa ineficaz de compensação, pois: i) aumento no tempo de pastejo é frequentemente interpretado como uma resposta compensatória dos animais frente a reduções na velocidade/taxa de ingestão de forragem ${ }^{(34)}$; ii) diminuição no tempo de ruminação pode ser consequência da diminuição na quantidade diária de material ingerido e, consequentemente, da menor quantidade de material a ser ruminado ${ }^{(35)}$; iii) como consequência dessa aparente restrição do processo de forrageamento e consumo diário, os animais apresentaram aumento no tempo destinado às atividades complementares. De fato, os dados de massa de forragem e de lâminas foliares (Tabela 2) e de comprimento de lâmina foliar (Figura 4) alicerçam a hipótese de um cenário restritivo ao processo de forrageamento. Ademais, devido à pequena magnitude de variação dos valores na composição química (Tabela 1), é pouco provável que os mesmos tenham gerado 
variações no comportamento ingestivo.

Tabela 5. Tempos de pastejo, ruminação e ócio de caprinos em pastos de capim-papuã mantidos em $10,20,30$ e $40 \mathrm{~cm}$ de altura sob lotação contínua

\begin{tabular}{|c|c|c|c|c|c|c|}
\hline \multirow{2}{*}{ Variável } & \multicolumn{4}{|c|}{ Tratamento $(\mathrm{cm})$} & \multirow{2}{*}{ EPM } & \multirow[t]{2}{*}{ Efeito } \\
\hline & 10 & 20 & 30 & 40 & & \\
\hline \multicolumn{7}{|c|}{ Ciclo 1} \\
\hline Pastejo & 519 & 445 & 445 & 424 & 16,3 & $\mathrm{~L}$ \\
\hline Ruminação & 195 & 213 & 255 & 350 & 20,0 & $\mathrm{~L}$ \\
\hline A. complementares & 726 & 782 & 740 & 667 & 26,0 & Q \\
\hline \multicolumn{7}{|c|}{ Ciclo 2} \\
\hline Pastejo & 387 & 489 & 428 & 419 & 21,2 & Q \\
\hline Ruminação & 227 & 253 & 340 & 346 & 21,9 & $\mathrm{~L}$ \\
\hline A. complementares & 826 & 698 & 672 & 675 & 37,4 & $\mathrm{~L}$ \\
\hline
\end{tabular}

Entretanto, os mecanismos descritos no parágrafo anterior parecem ter certos limites, uma vez que foram observadas respostas ligeiramente diferentes (i.e., desvios da linearidade) nos pastos mantidos em $10 \mathrm{~cm}$ (Tabela 5). Assim, no primeiro ciclo de avaliação, houve uma redução no tempo destinado às atividades complementares, provavelmente, em detrimento de um aumento no tempo de pastejo. Já no segundo ciclo, houve um aumento no tempo destinado às atividades complementares, provavelmente, em detrimento de uma diminuição no tempo de pastejo. Esse comportamento indica que com o transcorrer da exposição a um cenário bastante restritivo, e de uma tentativa inicial de compensação (ciclo 1), os animais passaram a relutar e/ou abdicar do pastejo (ciclo 2). Ribeiro Filho et al. ${ }^{(33)}$ e Rocha et al. ${ }^{(36)}$ reportaram comportamento semelhante com bovinos em pastos sob lotação intermitente, atribuindo tal situação a um baixo comprimento de lâminas residuais. Como descrito anteriormente, o valor limítrofe para a formação do bocado $(7-10 \mathrm{~cm})^{(16,32)}$ foi atingido quando os pastos foram mantidos com menos de $20 \mathrm{~cm}$ (Figura 4). Sendo assim, dentro das condições praticadas no presente estudo, pastos de papuã manejados com menos de $20 \mathrm{~cm}$ impõem um cenário extremamente restritivo ao processo de forrageamento e não devem ser adotados em nível de campo.

\section{Conclusão}

Os resultados aqui encontrados sugerem que pastos de papuã sob lotação contínua devem ser mantidos próximos de $40 \mathrm{~cm}$ quando o objetivo do sistema for assegurar a criação de estruturas acessíveis e de bom valor nutritivo durante todo o seu período de utilização.

\section{Referências}

1. Adami PF, Soares AB, Assmann TS, Assmann AL, Sartor LR, Pitta CSR, Franchin MF, Migliorini F. Dynamic of a papuã pasture under two grazing intensities and two nitrogen levels. R Bras Zootec. 2010; 39(12): 2569-2577. http://dx.doi.org/10.1590/S1516-35982010001200003 
2. Restle J, Roso C, Nörnberg JL, Brondani IL, Cerdótes L, Carrilho CO. Produção Animal em pastagem com gramíneas de estação quente. R Bras Zootec. 2002; 31(3): 1491-1500. http://dx.doi.org/10.1590/S1516$\underline{35982002000600021}$

3. Martins JD, Restle J, Barreto IL. Produção animal em capim papuã (Brachiaria plantaginea (Link) Hitchc) submetido a níveis de nitrogênio. Cienc Rural. 2000; 30(5): 887-892. http://dx.doi.org/10.1590/S0103$\underline{84782000000500025}$

4. Hodgson J, Clark DA, Mitchell RJ. Foraging behavior in grazing animals and its impact on plant communities. In: Fahey GC. Forage quality, evaluation and utilization. Lincoln: American Society of Agronomy; 1994. p.796-827.

5. Da Silva SC, Sbrissia AF, Pereira LET. Ecophysiology of C4 forage grasses - Understanding plant growth for optimising their use and management. Agriculture. 2015; 5(3): $598 \quad-\quad 625$. https://dx.doi.org/10.3390/agriculture5030598

6. Hodgson J, Da Silva SC. Options in tropical pasture management. In: XXXIX Reunião Anual da Sociedade Brasileira de Zootecnia, 2002. Anais... Recife: Sociedade Brasileira de Zootecnia; 2002. p.180-202.

7. Bircham JS, Hodgson J. The influence of sward condition on rates of herbage growth and senescence in mixed swards under continuous stocking management. Grass Forage Sci. 1983; 38(4): 323 - 331. https://dx.doi.org/10.1111/j.1365-2494.1983.tb01656.x

8. Da Silva SC, Gimenes FMA, Sarmento DOL, Sbrissia AF, Oliveira DE, Hernadez-Garay A, Pires AV. Grazing behaviour, herbage intake and animal performance of beef cattle heifers on marandu palisade grass subjected to intensities of continuous stocking management. J Agric Sci. 2013; 151(5): 727-739. http://dx.doi.org/10.1017/S0021859612000858

9. Alvares CA, Stape JL, Sentelhas PC, Gonçalves JLM, Sparovek G. Köppen's climate classification map for Brazil. Meterol Z. 2013; 22(6): 711-728. http://dx.doi.org/10.1127/0941-2948/2013/0507

10. EMBRAPA - Centro Nacional de Pesquisa de Solos. Sistema brasileiro de classificação de solos. 4 ed. Rio de Janeiro: Embrapa-SPI; 2014. 812 p.

11. CQFS - Comissão de química e fertilidade do solo. 2004. Manual de adubação e calagem para os estados do Rio Grande do Sul e de Santa Catarina, 10 ed. SBCS, Porto Alegre.

12. Sbrissia AF, Da Silva SC, Sarmento DOL, Molan LK, Andrade FME, Gonçalves AC, Lupinacci AV. Tillering dynamics in palisadegrass swards continuously stocked by cattle. Plant Ecol. 2010; 206(2): 349-359. https://dx.doi.org/10.1007/s11258-009-9647-7

13. Euclides VPB, Macedo MCM, Oliveira MP. Avaliação de diferentes métodos de amostragem para se estimar o valor nutritivo de forragem sob pastejo. R Bras Zootec. 1992; 21(4): 691-702.

14. Fonseca L, Mezzalira JC, Bremm C, Filho RSA., Gonda HL, Carvalho PCF. Management targets for maximising the short-term herbage intake rate of cattle grazing in Sorghum bicolor. Livest Sci. 2012; 145 (13): 205-211. https://dx.doi.org/10.1016/j.livsci.2012.02.003

15. Mezzalira JC, Carvalho PCF, Fonseca L, Bremm C, Cangiano C, Gonda HL, Laca EA. Behavioural mechanisms of intake rate by heifers grazing swards of contrasting structures. Appl Anim Behav Sci. 2014; 153 (April): 1 - 9. https://dx.doi.org/10.1016/j.applanim.2013.12.014

16. Lemaire G, Da Silva SC, Agnusdei M, Wade M, Hodgson J. Interactions between leaf lifespan and defoliation frequency in temperate and tropical pastures: a review. Grass Forage Sci. 2009; 64 (4): 341 - 353. https://dx.doi.org/10.1111/j.1365-2494.2009.00707.x

17. Silva VJ, Pedreira CGS, Sollenberger LE, Silva LS, Yasuoka JI, Almeida ICL. Canopy height and nitrogen 
affect herbage accumulation, nutritive value, and grazing efficiency of 'Mulato II' Brachiariagrass. Crop Sci. 2016; 56 (4): 2054-2061. http://dx.doi.org/10.2135/cropsci2015.12.0764

18. Chapman DF, Lee JM, Waghorn GC. Interaction between plant physiology and pasture feeding value: A review. Crop Pasture Sci. 2014; 65 (8): 721-734. https://dx.doi.org/10.1071/CP13379

19. Voltolini TV, Santos FAP, Martinez JC, Clarindo RL, Penati MA, Imaizumi H. Características produtivas e qualitativas do capim-elefante pastejado em intervalo fixo ou variável de acordo com a interceptação da radiação fotossintéticamente ativa. Rev Bras Zootec. 2010; 39 (5): 1002-1010. http://dx.doi.org/10.1590/S1516-35982010000500009

20. Niklas KJ. Plant allometry: the scaling process. 1 ed. Chicago: University of Chicago Press, 1994. 386 p.

21. Gregorini P, Gunter SA, Beck PA, Caldwell J, Bowman MT, Coblentz WK. Short-term foraging dynamics of cattle grazing swards with different canopy structures. J Anim Sci. 2009; 87(11): 3817-3824. http://dx.doi.org/10.2527/jas.2009.2094

22. Nantes NN, Euclides VPB, Montagner DB, Lempp B, Barbosa RA, Gois PO. Desempenho animal e características de pastos de capim-piatã submetidos a diferentes intensidades de pastejo. Pesq Agropec Bras. 2013; 48(1): 114-121. http://dx.doi.org/10.1590/S0100-204X2013000100015

23. Flores RS, Euclides VPB, Abrão MPC, Galbeiro S, Difante GS, Barbosa RA. Desempenho animal, produção de forragem e características estruturais dos capins marandu e xaraés submetidos a intensidades de pastejo. R Bras Zootec. 2008; 37(8): 1355-1365. http://dx.doi.org/10.1590/S1516-35982008000800004

24. Paula CCL, Euclides VPB, Montagner DB, Lempp B, Difante GS, Carloto MN. Estrutura do dossel, consumo e desempenho animal em pastos de capim-marandu sob lotação contínua. Arq Bras Med Vet Zootec. 2012; 64(1): 169-176. http://dx.doi.org/10.1590/S0102-09352012000100024

25. Da Silva SC, Nascimento Jr D. Avanços na pesquisa com plantas forrageiras tropicais em pastagens: características morfofisiológicas e manejo do pastejo. R Bras Zootec. 2007; 36 (suppl.0): 121-138. http://dx.doi.org/10.1590/S1516-35982007001000014

26. Black JL, Kenney PA. Factors affecting diet selection by sheep. II. Height and density of pasture. Aust J Agr Res. 1984; 35(4): 565-578. http://dx.doi.org/10.1071/AR9840565

27. Laca EA, Ungar ED, Demment MW. Effects of sward height and bulk density on bite dimension of cattle grazing homogenous swards. Grass Forage Sci. 1992; 47(1): 91-102. http://dx.doi.org/10.1111/j.13652494.1992.tb02251.x

28. Benvenutti MA, Gordon IJ, Poppi DP. The effect of the density and physical properties of grass stems on the foraging behaviour and instantaneous intake rate by cattle grazing an artificial reproductive tropical sward. Grass Forage Sci. 2006; 61(3): 272-281. http://dx.doi.org/10.1111/j.1365-2494.2006.00531.x

29. Benvenutti MA, Gordon IJ, Poppi DP. The effects of stem density of tropical swards and age of grazing cattle on their foraging behaviour. Grass Forage Sci. 2008; 63(1): 1-8. http://dx.doi.org/10.1111/j.13652494.2007.00609.x

30. Sollenberger LE, Burns JC. Canopy characteristics, ingestive behaviour and herbage intake in cultivated tropical grasslands. In: XIX International Grassland Congress, 2001. Proceedings... Piracicaba: Brazilian Sci. Anim. Husbandry; 2001 p.10-21. https:/www.internationalgrasslands.org/files/igc/publications/2001/tema82.pdf

31. Duru M, Ducrocq H. Growth and senescence of the successive leaves on a cocksfoot tiller. Effect of nitrogen and cutting regime. Ann. Bot. 2000; 85(5): 645-653. http://dx.doi.org/10.1006/anbo.1999.1117

32. Wade MH Factors affecting the availability of vegetative Lolium perenne to grazing dairy cows with 
special reference to sward characteristics, stocking rate and grazing method. Saint Gilles, 1991. 89p.These (Docteur) - Universite de Renne

33. Ribeiro Filho HMN, Setelich EA, Crestani S, Dias KM, Mantovani C, Valenti J. Relationship between diurnal grazing time and herbage intake in dairy cows in rotational grazing. Cienc Rural; 2011. 41(11): 20102013. http://dx.doi.org/10.1590/S0103-84782011005000140

34. Da Silva SC, Carvalho PCF. Foraging behavior and herbage intake in the favorable tropics/subtropics. In: McGilloway. Grassland: a global resource. Wageningen: Wageningen Academic Publishers; 2005. p. 81-96.

35. Welch JG, Smith AM. Influence of forage quality on rumination time in sheep. J Anim Sci. 1969; 28(6):813-818. https://dx.doi.org/10.2527/jas1969.286813x

36. Rocha CH, Santos GT, Padilha DA, Schmitt D, Medeiros-Neto C, Sbrissia AF. Padrões de deslocamento de bovinos em pastos de capim-quicuiu sob lotação intermitente. Arq Bras Med Vet Zootec. 2016; 68(6): 1647 1654. http://dx.doi.org/10.1590/1678-4162-8711 